\title{
Control, monitoring and warning computerized system for a semi-automated sliding system of assembled roof
}

\author{
Shigeomi Nishigaki ${ }^{1}$ \\ Yoshio Maruyama ${ }^{2}$ \\ Osami Matsunaga ${ }^{3}$ \\ Yoshinobu Futamura $^{4}$ \\ Seisaku Kato ${ }^{5}$ \\ Hiromi Fukuchi ${ }^{6}$ \\ Shoshi Kazue ${ }^{7}$
}

\begin{abstract}
Faced by a need to overcome work force shortage and vanishing skill replacement, to avoid dangerous works and to reduce cost, a semi-automated sliding system of assembled roof is developed to build a roof of machine room within underground power plant. The semi-automated sliding system will improve working conditions, since it performs certain repetitive dangerous operation without fatigue and errors that often occur when workers perform these tasks. Of necessity here is a control, monitoring and warning computerized system to monitor the behavior of the assembled roof being slid; check whether its behavior is approaching its safety operating limits under operations; and automatically stop its behavior in emergency. First, this paper describes problems and motive in this study. Secondly, presented in detail is the implementation of the monitoring and warning computerized system being developed. Finally, the field evaluation and remarks are reported.
\end{abstract}

\section{1: Problems and Motive}

Faced by a need to overcome work force shortage and vanishing skill replacement, to avoid dangerous works and to reduce cost, a semi-automated sliding sy stem of assembled roof is developed to build a roof of machine room within underground power plant. Figure 1 presents the roof of machine room being constructed.

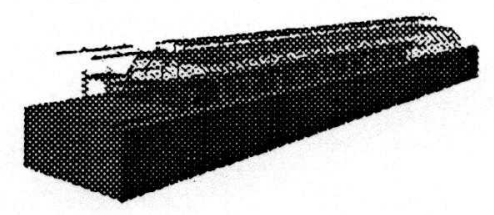

Figure 1: The Roof of Machine Room being Constructed

\footnotetext{
' Senior Research Engineer, Technical Research Institute, Hazama Corporation,

515-1 Nishimukai Karima, Tukuba, Ibaraki 305, Japan, email: MAD02724@niftyserve.or.jp

${ }^{2}$ Research Engineer, Technical Research Institute, Hazama Corporation,

515-1 Nishimukai Karima, Tukuba, Ibaraki 305, Japan, email: MHE02446@niftyserve.or.jp

${ }^{3}$ Engineer, Oku-Tataragi Office, Hazama Corporation, 156-1 Hibara Tataragi, Asago-cho, Asago-gun, Hyogo 679-34, Japan, email: HZM00413@niftyserve.or.jp

${ }^{4}$ Manager, Oku-Tataragi Office, Hazama Corporation, 156-1 Hibara Tataragi, Asago-cho, Asago-gun, Hyogo 679-34, Japan, email: HZM00931@niftyserve.or.jp

${ }^{5}$ General Manager, Oku-Tataragi Office, Hazama Corporation, 156-1 Hibara Tataragi, Asago-cho, Asago-gun, Hyogo 679-34, Japan

${ }^{6}$ Engineer, Okutataragi Power Plant Extension Project Office, The Kansai Electric Power Co., Inc. 740-1 Aza Ogura Tataragi, Asago-cho, Asagogun, Hyogo 679-34, Japan

${ }_{7}^{7}$ Manager, Okutataragi Power Plant Extension Project Office, The Kansai Electric Power Co., Inc. 740-1 Aza Ogura Tataragi, Asago-cho, Asago-

gun, Hyogo 679-34, Japan
} 
First, Each steel is brought in the underground work site. Second, the each steel frame is pre-assembled at onsite yard. Third, the assembled one is lifted up, transported, placed and connected to the existing roof by girders and braces. Fourth, the assembled roof with wheels on rails is pushed forward at distance of $80 \mathrm{~cm}$ in one stroke operation by two hydraulic jacks. The stroke operation is subsequently repeated five times. In consequence, the assembled roof is moved forward at distance of four meters in a workshift. A sequence of these construction operations is repeated on a basis of eight-days cycle. The repetition of the sequence results in building a roof of underground power plant as shown in Figure 1.

The particular requirements in operating the semiautomated sliding system of assembled roof are summarized in Figure 2.

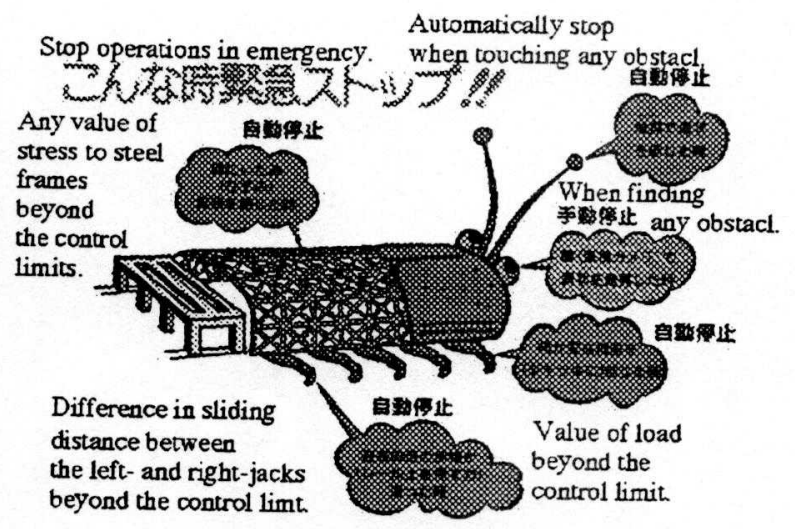

Figure 2: The Requirements in Operating the Semiautomated Sliding System

The point here is that gagging and forcing functions are available to avoid any collision of interference and any excessive load by the two hydraulic jacks to damage the structural elements of the assembled roof. The gagging function works to prevents workers from conducting irregularintentions. The forcing function works to prevent the behavior from continuing until the problem has been corrected.

The semi-automated sliding system will improve working conditions, since it performs certain repetitive dangerous operation without fatigue and errors that of ten occur when workers perform these tasks. Of necessity here is a control, monitoring and waming computerized system to:

- monitor the behavior of the assembled roof being slid, - check whether its behavior is approaching its safety operating limits under operations, and

- automatically stop its behavior in emergency.

With the control, monitoring and warning computerized system, workers may perform the steps as follows:

Step 1: System start-up

- Examine system components and instrument readings to ensure that the components are readily to be put on-line.

- Once this has been done, the operators follow standard operating procedure for start-up.

Step 2: System monitoring

- Monitor its performance.

- Key process variables monitored or sensed at all points include temperature, pressure, flow rate, and so on.

- This information is continually available at computer terminals.

Step 3: Response to warnings

- In the case of system upset or equipment malfunction, warnings warn operators of the need for their intervention.

- The operators are trained to recognize warning signals and to know the appropriate warning responses.

Step 4: Shutdown of system components

Step 5: Routine maintenance. If necessary, request maintenance assistance.

Work operations here are more likely to relate to intervention, preventive maintenance, or test and replacement of components. Work operations shift from physical to increased cognitive workload in with CRT interface.

Human error can be categorized into four basic types: lapse, slip, mistake and violation [1]. Lapse is a memory failure, for example, forgetting to remember to carry out intended actions at the appropriate time and place such as omitting planned items, place-losing, and forgetting intention. Slips is execution failure. It means that the intentions were appropriate enough, but the actions were not executed as planned. Mistake is planning or procedure failure. It means that the actions may be carried out as planned, but the plan is inadequate to achieve its desired outcome. Violations may be categonized into routine violations and exceptional ones. The routine violations are largely habitual, forming an established part of an individual's behavior repertoire. The exceptional violations are singular violations occurring in a particular of circumstances, for example, particular tasks or operating circumstances that make violations inevitable, no matter 
how well-intentioned the workers might be.

It has been suggested that there are three cognitive levels, namely skill-, rule- and knowledge-based levels, involved in conceiving and then carrying out an action sequence ([1] [2]). Behavior or performance at these three levels correspond to a decreasing in the degree of familiarity with the environment or the tasks. Slips and lapses at the skill-based level generally precede the detection of a problem, but mistakes and violations at the rule-based and knowledge-based levels often occur after detecting a problem and during subsequent attempts to find its solution [2].

Behavior at the skill-based level represents reflexive, well-rehearsed motor skill behavior (e.g., skill of the craft) in familiar surroundings. The information at skill-based level is in the form of signal, as cues triggering the appropriate actions, that is continuos quantitative indicator of the time-space behavior of the environment and is derived at a specific point in time. Behavior at skill-based level rolls along without conscious attention or control [1], and is govern by stored patterns of pre-programmed instructions, i.e., If < action $>$ then $<$ reaction $>$ or If < activator $>$ then $<$ activate $>$. The condition parts are composed of recency or frequency of previous successful use or experience and environmental control of signals.

At the rule-based level, behavior follows rule or procedure selected from previous successful experiences. Behavior at rule-based level is guided by signs relating to stored rules or procedures in the forms of If $<$ situation $>$ then $<$ state $>$ or If $<$ state $>$ then $<$ remedial actions $>$. Signs here refer to names being labeled to situations or states by convention or prior experience. Behavior here is goaloriented based on feedforward control by stored rules or procedures. When a conscious check in progress of work detects a problem, the rules and procedures are applied to solve the problems by survival of the fittest.

Knowledge-based behavior is evoked when entirely new, unstructured, or complex problems are encountered. In unfamiliar, behavior tends to be goal-driven in the sense that a successful or wrong sequence of the actions is selected based on the attempts made to attain the desired outcome. At the knowledge-based level, a task is accomplished by setting goals, initiating actions to achieve them, observing the extent to which the actions are successful and then modifying them to minimize the discrepancy between the present situation and the desired state. A strategicmistake occurs when selecting the wrong goal. A tactical mistake happens when taking the wrong path. Symbols refer to concepts tied to functional properties and can be used for reasoning and computation by means of a suitable representation of such properties [1]. Behavior at knowledge-based level could be guided by symbols to search for suitable analogies, or diagnostic theory in order to fit the current situation such as If $<$ It's like the situation $\mathrm{X}>$ then $<\mathrm{I}$ should try action $\mathrm{Y}>$.

Since the semi-automated sliding system is composed of many sub-systems and has a great variety of rules and procedures for operations, in looks andfeel, it is so complex and a blackbox to workers. Workers, who operate the semi-automated sliding system, are waiting for somewhat like happenings during operations along as expecting nothing may happen. Workers may not, however, pay their full attention to everything all along. Sensors attached with the semi-automated construction system will provide a large amount of single sensor type information grouped either functionally, by sequence, or by hierarchy. Absent-minded slips or lapses at skill-based level may often cause an omission of necessary inspections. When confronted with a problem, workers are more likely to use a prepackaged and well-rehearsed solution at the rule-based level than resorting to a knowledge-based level. Lack of details or limited procedures may shift workers from a ruleor procedure-supported level to the-seat-of-the-pants approach. Since workers' reasoning mechanism may have tunnel view and channeled attention according to the degree of partial understanding, floods of information often go beyond their ability to digest. Mistakes at rule-based level are more likely to arise from problems with memory, lack of willingness to check each step in a procedure, and the like. In unfamiliar situations when proven rules are not available or workers run out of their stockpile of rules and procedures, behavior may be goal-oriented in the sense that different attempts are made to attain the desired outcome, and a successful or wrong sequence of the actions is then selected.

This study centers on:

At skill-based level: Making the boundaries of acceptable performance of the semi-automated sliding system visible to the workers;

A t rule-based level: Representing cues for actions not only as readily understandable signs, but also indicating the preconditions of the validity of actions; and

At knowledge-based level:

(1) Providing a mimic diagram as an overview display wherein information is summarized as symbols to support functional understanding during problemsolving; and

(2) Providing support to analyze the observed data to present information on the existing state of operations to:

(a) judge whether the transient state of the monitored process is approaching its safety operating limits; and

(b) find of values of control limits suitable to avoid the damage of the structural elements of the assembled 
roof while keeping the availability of the semiautomated sliding system.

\section{2: Implementation}

\section{1: Schematic View}

Figure 3 shows the schematic view of the control, monitoring and warning computerized system being implemented in the actual site. The key process variables in Figure 4 represent the transient state of the particular requirements shown in Figure 2. The key process variables should be monitored or sensed at all points in time under operations.

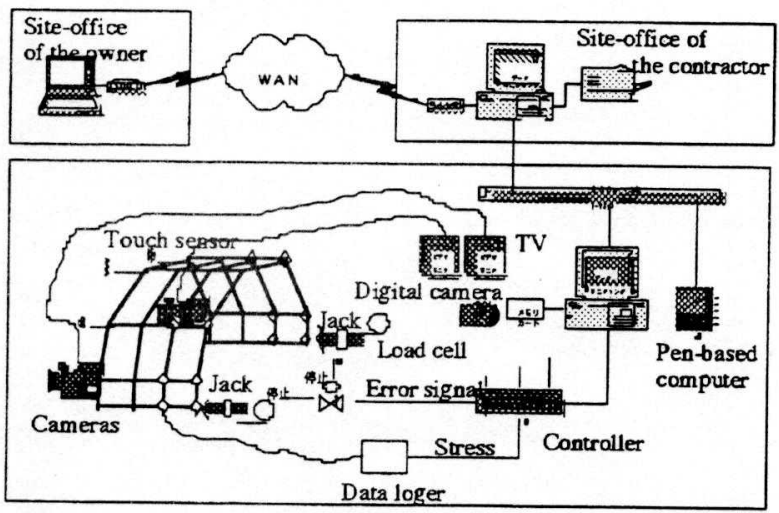

Figure 3: The Schematic View of the Monitoring and Warning Computerized System

\begin{tabular}{|l|l|}
\hline \multicolumn{1}{|c|}{ Key Process Variables } & $\begin{array}{c}\text { Sensor } \\
\text { a b c d e f }\end{array}$ \\
\hline (1) On/off information of interference & \\
(2) Effective stress of each component of roof & (3) Measure pressure by each jack loaded on roof to avoid overload. \\
(4) Each sliding distance & \\
(5) Difference between left sliding distance and right one & \\
(6) Sliding speed & \\
(7) Digital picure & \\
(8) Flashing red light with buzzer to indicate emergency &
\end{tabular}

Legend: a: Touch sensor, b: Strain gage, c: Load cell. d: Encoder. e: Camera. f: Light

Figure 4: Key Process Variables

\section{2: Signals, Signs and Symbols}

Figure 5 shows the relationship through signal, sign and symbol between the key process variables and the recovery functions being built in the control, monitoring and warning computerized system.

The signal is continuous quantitative indicator of the time-space behavior of the semi-automatic sliding system. The signals are categorized into error signals and control signals. The error signals represent the differences between the actual state and the intended or planned state in a timespace behavior. The control signals shows the transient state of behavior at a specific point in time. The boundaries of acceptable performance of signals are inputted into the control, monitor and warning computerized system.

Because workers have to rely on the displayed signals that stem from sensors, required are appropriate signs with proactive advises for retention of a conscious check in progress of work and for application of suitable rules or procedures. Any value of signal beyond the control limit values evokes a built-in self-stopping function and displays a sign which interprets a currently critical situation. Signs are labeled by names of states or situations of the semiautomatic sliding system. Signs, which are build in IFTHEN rules, are used to select or activate stored predetermined actions that control the sequence of routine operations.

Workers can understand the existing state of the system by control signals and off-normal state by error signals. They can select and activate predetermined recovery actions by signs. As previously mentioned, symbols refer to concepts tied to functional properties and can be used for reasoning and computation by means of a suitable representation of such properties. Information is treated as symbols that could be directly utilized and manipulated within the mimic diagram as presented later. Furthermore, workers can reason some potential faults based on symbols displayed in the mimic diagram, as confirming and asking for additional data.

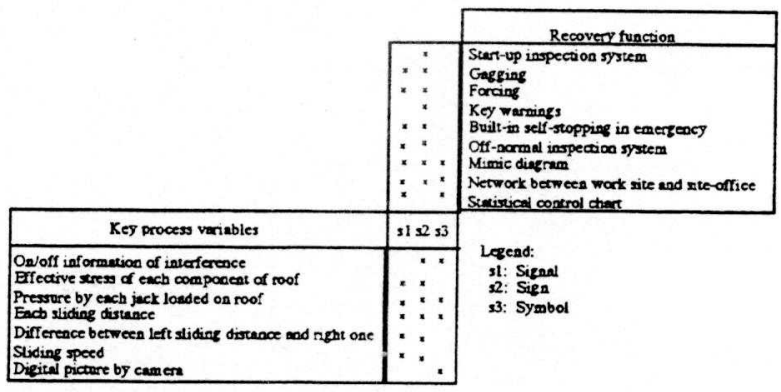

Figure 5: the Key Process Variables and the Recovery Functions

\section{3: Start-up inspection}

The start-up inspection system is a pen-based computer system to display a list of items to be checkedin order to ensure that the system components are readily to be put on-line and no any obstacle in the environment. Unless the inspections results by the start-up inspection system are loaded up to the control, monitoring and 
warnings computerized system, the operation of the two hydraulic jacks can not start to be pushed forward.

\section{4: Built-in self-stopping}

Any value of signal beyond the control limit values automatically shut down jack operations and inevitably display key warnings. The key warnings mean that the warnings in questions are judged by experts to be very important for workers to identify faults and to correct them. The key warnings will be provided as a decision aid when confronted off-normal event, for examples, when value of the each key variable go out of the control limits.

\section{5: Off-normal inspection}

Workers have to take predetermined recovery actions or manipulations under off-normal situations instead of first resetting a button. The off-normal inspection system is a pen-based computer system to display a list of items to be checked in off-normal situations. Unless inspections results by the off-normal inspection system are up-loaded to the control, monitoring andwarnings computerized system, the operations of the two hydraulic jacks can not re-start to be pushed forward.

\section{6: Gagging, forcing and memory Aid}

To overcome a failure to attend any change or countersign in operations, gagging and forcing functions are built in the control, monitoring and warning computerized system. Memory aid is to provide an interactive checklist facility so that appropriate actions could be taken on a timely basis. Both the start-up inspection system and the offnormal inspection system work as gagging, force memory aid functions to provide a set of procedures that not only give workers a step-by-step guidance in the do's and the don'ts but also prompt them to check whether easily omitted steps have been completed.

\section{7: Mimic diagram}

As mentioned earlier, a great variety and volume of information on key process variables of ten go beyond an worker's experience and ability to digest. It is important that workers could pick a set of building block of information at the right level of abstraction and increase their situational awareness. Therefore, information on key process variables is summarized into a mimic diagram in Figure 6.

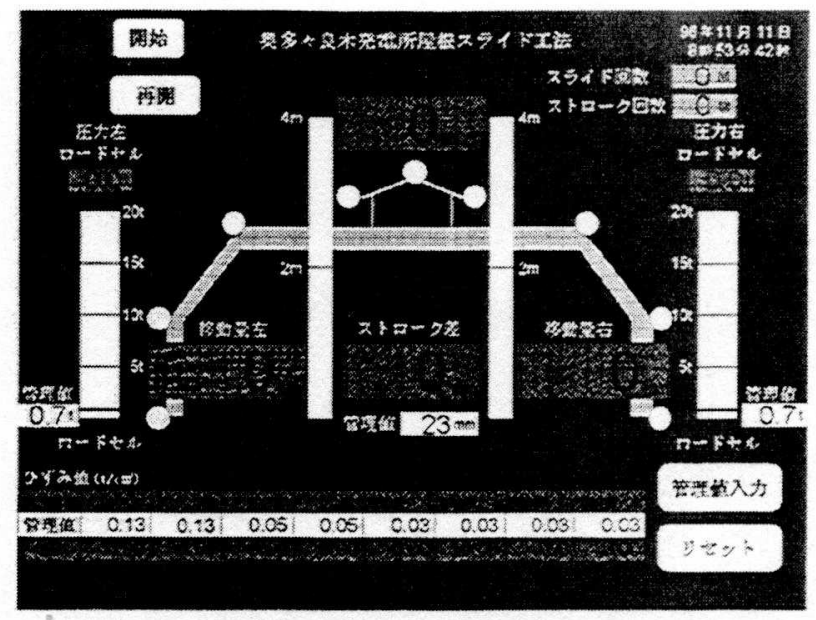

Figure 6: The mimic diagram of The Control, Monitoring and Warnings Computerized System

The mimic diagram delineates schematic overview of the semi-automated sliding system and represents the physical topography of system performance in a time-space behavior. The alphanumeric information display movement values of eachjack at a stroke, the number of times of sliding, each value of each effective stress, etc. are shown in the mimic diagram. These values are very important for workers to judge whether or not movement of each equipment at a stroke is synchronized with others and is approaching its safety operating limits. In off-normal case, not only key warnings in sentence but also the topographic representation of the off-normal place will be displayed on the mimic diagram. The mimic diagram will provide workers with information as symbols of use to topographically investigate improper function and to symptomatically reason its underlying causes.

\section{8: Network between Work-Site and Site-offices}

All sensed data and digital pictures of construction operations in progress can be up-loaded through ethernet from the control, monitoring and waming computerized system at the work site to the thinking and decision-support system equipped with the site office of the contractor. The thinking and decision-support system is composed of planning and scheduling system integrated with $3 \mathrm{D}$ model of the assembled roof and a statistic analysis system. The site-office is located at a distance of about 1,500 meters from the work site. Site-manger and engineers could monitor and analyze all the sensed data by the thinking and decision-support system. To augment the limited capacity of their working memory, the thinking and decision-support system serves two primary functions:

(a) as a working database wherein analytical operations can 
be performed; and

(b) as a means of keeping track of progress of construction operations by relating current data to plan and scheduling stored in the knowledge-base.

Manager and engineers at the owner-office can remotely access the working database and the knowledge-base.

By analyzing all the sensed data, site-manager and engineers understand trends in the key process variables and ensure whether or not the system is approaching a safety control limit.

\section{3: Field Evaluation and Practical Problems}

At the end of October 1996, the control, monitoring and warning computerized system being developed was implemented with the semi-automated sliding system of assembled roof in an actual work site to build a roof of machine room within underground power plant. First, conducted were several field tests to confirm the availability of the eachfunction of the control, monitoring and warning computerized system. Under the field tests, the control, monitoring and warning computerized system could correctly capture signals from the sensors; the built-in self stopping function was evoked as designed by the excessive load beyond the control limit values, which were caused by a tool being experimentally left on the rail and, in another case, were stemmed from workers unexpectedly on the jobs of inspecting the assembled roof. The semi-automated sliding system has been and is being operated since the beginning of November, 1997. So far sliding operations of ten times have been conducted effectively, that is, safely and efficiently.

An engineer at the site-office intermittently analyzes the observed data, and find the suitable values of control limits and input them into the control, monitoring and warning computerized system in attempting to deal with steps as shown in Figure 7.

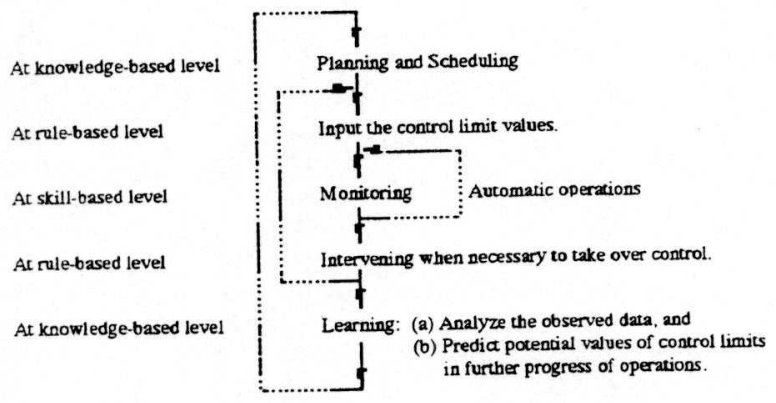

Figure 7: Steps to Update values of Control Limits
As explained in the section of problems and motive, five stroke operations are conducted in a workshift, and further sliding the assembled roof is repeated 25 times at eight-day cycle. Capturing signals at interval of three seconds from the load cells inevitably provides a large amount of the observed data. From the practical view point, it is very important to find values of control limits of loads to the two hydraulic jacks. The lower values of the control limits will frequently evoke the built-in self-stopping function and result in the poor constructability. On the contrary, the higher values inevitably allow excessiveloads to damage the structural elements of the assembled roof.

The Type 1 extreme values distribution is applied to decide the control limit values. In practice, first selected are the upperfive values in decreasing order of the observed data by the each stroke and in turn the 25 sample data can be obtained. Second, calculated are the scale parameter " $a_{i}$ " and the characteristic largest value " $w_{i}$ " of the Type 1 extreme value distribution corresponding to the given sample data obtained in " $\boldsymbol{i}$ th" workshift; $\boldsymbol{i}=1,2, \cdots, I$, where " $I$ " denotes the current workshift. The average $" \bar{x}_{i}$ " and the standard deviation $" s_{x_{i}}$ " are given by $\bar{x}_{i}=w_{i}+\frac{\gamma}{a_{i}}$ and $S_{x_{i}}=\frac{\pi}{\sqrt{6} a_{i}}$, respectively, where " $\gamma$ " denoted the Euler's constant. Third, by applying the least square method to the given data " $\bar{x}_{i}$ " and " $s_{x_{i}}$ "; $i=1,2, \cdots, I$, the predicted values of the " $\bar{x}_{i}$ " and " $s_{x_{i}}$ "; $i=I+1, \cdots, 25$ can be obtained. The given data and predicted values with respect to the " $\bar{x}_{i}$ " and " $s_{x_{i}}$ " are shown in Figure 8, respectively.
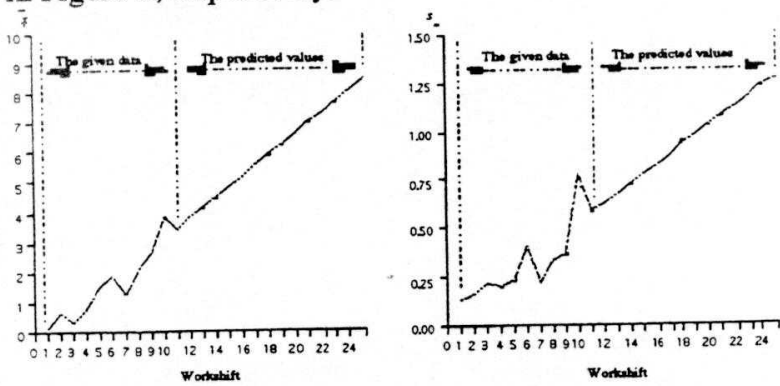

Figure 8: The Given Data and The Predicted Values of the " $\bar{x}_{i} "$ and the $" s_{x_{i}} "$

Now the scale parameter " $\hat{a}_{i}$ " and the characteristic largest value " $\hat{w}_{i} ; i=I+1, \cdots, 25$ are predicted by $\hat{a}_{i}=\frac{\pi}{\sqrt{6} \hat{s}_{x_{i}}}$ and $\hat{w}_{i}=\bar{x}_{i}-\frac{\sqrt{6}}{\pi} \gamma \hat{s}_{x_{i}}$, respectively. By the Type 1 extreme distribution with the " $\hat{a}_{i}$ " and " $\hat{w}_{i}$ "; 
$i=I+1, \cdots, 25$, predicted is the probability that extreme values may be emergent in the remaining workshifts. The each control limit value for the remaining workshifts can be determined at the point of $99 \%$ probability of the Type 1 extreme value distribution corresponding to the given workshift. Figure 9 presents the Type 1 extreme value distributions corresponding to the 15 th workshift and the 20 th workshift.

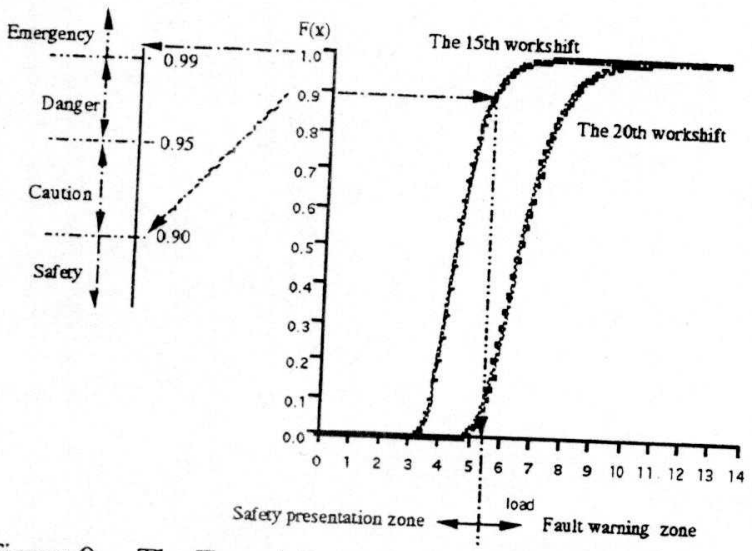

Figure 9: The Type 1 Extreme Value Distributions corresponding to the 15 th Workshift and the 20 th Workshift

Based on the analysis results by the method as discussed above, we will be able to give the site manager; the engineers and the shift supervisor the interpretation of data and possible courses of construction operations. The operation of the semi-automated sliding system is scheduled to end at the beginning of June, 1997. Then we will be able to confirm the validity of the method as discussed above.

\section{References}

[1] J. Rasmussen (1983). Skills, Rules, and Knowledge; Signals, Signs, and Symbols, and Other Distinctions in Human Performance Models, IEEE Transactions on Systems, Man, and Cybernetics, Vol. SMC-13, No. 3, 257-266.

[2] J. Reason (1990). Human Error, Cambridge University Press, New York

[3] D. Petersen (1982). Human-Error Reduction and Safety Management, Garland STPM Press, New York.

[4] J. Reason (1987). "Generic Error-Modeling System (GEMS): A Cognitive Framework for Locating Common Human Error Forms." Rasmussen, J., Duncan, K. and Leplat, J. edited: New Technology and Human Error, John Wiley \& Sons, pp. 63-83

[5] S. S. Rao (1992). Reliability-Based Design, McGraw-Hill [6] A. H-S. Ang and W. H. Tang (1988). Probability Concepts in Engineering Planning and Design, John Wiley \& Sons.
[7] S. Nishigaki, K. H. Law (1994). Safety Problems in Onsite Construction Work Processes, Proceedings of the 11 th International Symposium on Automation and Robotics in Construction (ISARC), Brighton, U. K., 13-18 